Research Article

\title{
Fault-Tolerant Control for Three-Tank System in Case of Sensor Faults
}

\author{
Mondher Amor (D), Taoufik Ladhari $(D$, , Salim Hadj Said $(D$, and Faouzi M'Sahli \\ Industrial Systems Study and Renewable Energy Unit, National Engineering School of Monastir, University of Monastir, \\ Ibn El Jazzar Street, Monastir 5019, Tunisia \\ Correspondence should be addressed to Mondher Amor; mondheramor@yahoo.fr
}

Received 4 September 2020; Revised 17 December 2020; Accepted 2 January 2021; Published 18 January 2021

Academic Editor: José Antonio Rosales Martínez

Copyright (c) 2021 Mondher Amor et al. This is an open access article distributed under the Creative Commons Attribution License, which permits unrestricted use, distribution, and reproduction in any medium, provided the original work is properly cited.

\begin{abstract}
This research paper would be devoted to the application of a fault-tolerant control (FTC) for a benchmark system composed of three interconnected tanks in case of sensor faults. The control scheme includes two blocks: fault detection and isolation (FDI) block and a control law reconfiguration block. The strategy of the FDI method is based on a bank of high gain observers; each of them is constructed to estimate the system state vector. Thus, the diagnostic signal-residuals are generated by the comparison of measured and estimated outputs and the faulty sensor is isolated. The reconfiguration block performs an update of the controller parameters according to the operating mode. The application of this method to a pilot plant demonstrates that the hydrographic system maintains quite performances after sensor faults occurrence.
\end{abstract}

\section{Introduction}

Any automation of a process aims at reaching an almost perfect solution to obtain a final product of good quality and health of all faults. The automated system control theory has been widely developed and applied to the industrial process. These techniques ensure the stability of the closed-loop system and yield a predefined performance in the case where all system components operate safely. However, the more automated the process is, the more it is subject to fault occurrence. Hence, the need for a control method that is able to ensure nominal performance within highly automated systems where immediate maintenance is out of reach. This control is referred to as fault-tolerant control (FTC) which becomes extremely important in the last few decades. There are two approaches to the synthesis of an FTC. One approach, known as passive FTC, aims at designing a robust controller against some given faults. Another approach, known as active FTC, requires a fast fault detection and isolation (FDI) algorithm followed by a control law adjustment that allows maintaining high performances in the controlled system.
The detection and isolation of faults is an important research area in process control due to the improvements that can be reached in terms of the safety and reliability of the plant. This can be traced from some valuable survey papers [1-3] and books $[4,5]$. Different methods have been developed and implemented in different directions and for several systems [6-11] such as model-based method $[12,13]$, observer method [14-16], parameter estimation method [17], parity space method [18], and a combination of these methods with artificial intelligent $[8,19]$.

The three-tank system (3TS) is considered an important and effective prototype of many applications in industrial processes, such as water treatment, food industry, chemical and petrochemical plants, oil, and gas systems. It is widely used in water conditioning systems, which provide the user with an abundant supply of luxuriously conditioned water and in craft brewing systems. In spite of the fact that many fault detection and isolation methods have already been applied to three-tank system systems, a few fault accommodation techniques have been considered. In [20], a feedback linearization approach for fault-tolerant control in a 3TS benchmark is investigated. Noura et al. proposed an 
approach based on the online estimation for the fault and the computation of additive control law is able to compensate for the fault effect on the system [21]. Mendoca et al. have used predictive control and fuzzy logic to design a faulttolerant control for a 3TS [22]. Orani et al. presented a global observer based on a second-order sliding mode control algorithm for the simultaneous fault detection, isolation, and reconstruction for hydraulic vertical 3TS [23]. Other researchers have focused on fault diagnosis and accommodation for both sensor and actuator faults; they have proposed an analytical redundancy method to solve the drawbacks of the hardware redundancy such as cost and space [24]. It should be noted that all these previous works have developed FTC solutions based on the linearized model of the 3TS. This means that these approaches are valid only around an operating area. To overcome the previous drawbacks, some papers have focused on the application of actuator fault magnitude estimation [25] or fault-tolerant control [26-28] by using a nonlinear model of the 3TS.

In this paper, we focus on the online sensor fault detection and isolation by adopting high gain observers' bank. A reconfiguration of the controller is then performed by adjusting the design parameters to compensate for the sensor fault effects. Compared to previous works, the proposed approach has the feature to potentially mitigate the required time of the FDI process as well as the ability to reach the reference trajectory after the fault occurrence. The other purpose of this paper is to show the experimental performance of the proposed FTC method on a real plant. Moreover, it is important to mention that the proposed FTC approach is valid for several working areas and not only locally around the operating point.

The paper is organized as follows. Section 2 presents the 3TS model to illustrate sensor faults and accommodation methods. The next section is devoted to the strategy of fault diagnosis based on high gain observers bank used to detect, isolate, and estimate the faults. In section four, the full faulttolerant control scheme is exposed. In this context, a new adjusted control law that aims to reduce the fault effect on the system outputs is discussed. Experimental results are given and presented in section 5. Finally, a conclusion and some perspectives are given in the sixth section.

\section{Three-Tank System Presentation}

2.1. Plant Description. The 3TS plant consists of three identical cubes with the same cross section area $S$. These tanks are coupled serially to each other via cylindrical pipes of the same cross section area $S_{n}$. The complete structure of the plant is shown in Figure 1.

Two pumps $P_{1}$ and $P_{2}$, driven by DC-motors, represent, respectively, the input flows $Q_{1}$ and $Q_{2}$ of tanks $T_{1}$ and $T_{3}$. The plant is a closed system, in which the liquid that enters the reservoir from the tanks returns to the tanks thanks to two pumps.

Besides the outflow valve on $T_{3}$, the system includes five additional valves. Two of them are used to join each pair of neighboring tanks and can be manually tuned to close the connection between the two consecutive tanks. The other three valves $V_{11}, V_{12}$, and $V_{13}$ are at the bottom of each tank.

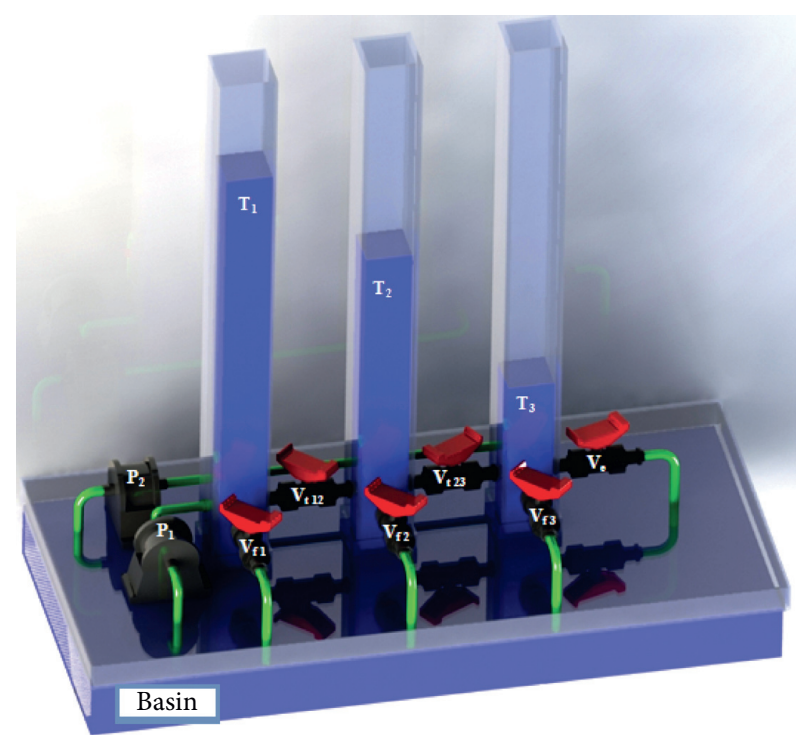

Figure 1: Full structure of the computer design plant.

These leak valves can be used to manually drain each tank [29]. A piezoresistive differential pressure sensor, associated with each tank, delivers an analog voltage signal to measure the three liquid levels denoted by $h_{1}, h_{2}$, and $h_{3}$.

2.2. Mathematical Model. The analytic model can be easily derived from the principle of mass conservation and the Torricelli law. In fact, the change of water volume in tank $i$ $(i=1: 3)$ is determined by the following equation:

$$
\dot{V}_{i}=S_{i} \cdot \frac{\mathrm{d} h_{i}}{\mathrm{~d} t}=\sum Q_{\mathrm{in}, i}-\sum Q_{\mathrm{out}, i},
$$

where $\sum Q_{\mathrm{in}, i}$ and $\sum Q_{\mathrm{out}, i}$ represent the total liquid inflows and outflows in tank $i$, respectively. Then, the mathematical model is specified by the following mass balance equations:

$$
\left\{\begin{array}{l}
\dot{h}_{1}(t)=\frac{1}{S}\left(Q_{1}(t)-Q_{12}(t)-Q_{l 1}(t)\right), \\
\dot{h}_{2}(t)=\frac{1}{S}\left(Q_{12}(t)-Q_{23}(t)-Q_{l 2}(t)\right), \\
\dot{h}_{3}(t)=\frac{1}{S}\left(Q_{2}(t)+Q_{23}(t)-Q_{e}(t)-Q_{l 3}(t)\right),
\end{array}\right.
$$

where $t$ represents the time; $h_{1}, h_{2}$, and $h_{3}$ represent the liquid levels in each tank; $S$ represents the cross section of the tanks; $Q_{1}$ and $Q_{2}$ designate respectively the flow rates of pumps $P_{1}$ and $P_{2} ; Q_{i j}$ denotes the flow rates between tank $T_{i}$ and $T_{j}$; and $Q_{l i}$ represents the output flow of the corresponding tank when its leak valve is open. $Q_{e}$ is the leakage valve. The flows $Q_{i j}$ and $Q_{e}$ in (2) are given by Torricelli's law as follows:

$$
\begin{aligned}
Q_{i j}(t) & =a_{z i} S_{n} \operatorname{sgn}\left(h_{i}-h_{j}\right) \sqrt{2 g\left|h_{i}-h_{j}\right|}, \\
Q_{e}(t) & =a_{z 3} S_{n} \sqrt{2 g h_{3}},
\end{aligned}
$$


where $a_{z i}$ is the outflow coefficient, $\operatorname{sgn}($.$) is the sign of the$ argument, and $g$ is the acceleration of gravity. Consequently, the nonlinear $3 \mathrm{~T}$ model is given as follows:

$$
\left\{\begin{array}{l}
\frac{\mathrm{d} h_{1}}{\mathrm{~d} t}=-a_{1} \operatorname{sign}\left(h_{1}-h_{2}\right) \sqrt{\left|h_{1}-h_{2}\right|}+\frac{Q_{1}}{S}, \\
\frac{\mathrm{d} h_{2}}{\mathrm{~d} t}=a_{1} \operatorname{sign}\left(h_{1}-h_{2}\right) \sqrt{\left|h_{1}-h_{2}\right|}-a_{2} \operatorname{sign}\left(h_{2}-h_{3}\right) \sqrt{\left|h_{2}-h_{3}\right|}, \\
\frac{\mathrm{d} h_{3}}{\mathrm{~d} t}=a_{2} \operatorname{sign}\left(h_{2}-h_{3}\right) \sqrt{\left|h_{2}-h_{3}\right|}-a_{3} \sqrt{h_{3}}+\frac{Q_{2}}{S},
\end{array}\right.
$$

where $a_{i}$ represents the system parameter given by

$$
a_{i}=\frac{1}{S} a_{z i} S_{n} \sqrt{2 g} ; \quad i=1, \ldots, 3 .
$$

2.3. Three-Tank System Faults Representation. The 3TS laboratory system is considered as a rich ground to serve as a test environment for the FTC. It is used as a benchmark system that can be affected by various additive and/or multiplicative faults:

(i) Faults actuator: an actuator fault can be represented by

$$
u_{i}^{f}(k)=\alpha_{i} u_{i}(k)+u_{i 0} ; \quad i=1,2,
$$

(i) where $u_{i}^{f}$ and $u_{i}$ represent the faulty and the normal control action of the $i$ th pump, respectively. The constant offset is denoted by $u_{i 0}$ and $0 \leq \alpha_{i} \leq 1 \mathrm{de}-$ notes a gain degradation of the $i^{\text {th }}$ actuator.

(ii) Faults sensor: similar to the actuator fault representation, a faulty output can be written as

$$
y_{j}^{f}(k)=\beta_{j} y_{j}(k)+y_{j 0} ; \quad j=1,2,3,
$$

where $y_{j}^{f}$ and $y_{j}$ represent the faulty and the normal level of the $j^{\text {th }}$ sensor, respectively. The constant offset is denoted by $y_{j 0}$ and $0 \leq \beta_{j} \leq 1$ denotes a gain degradation of the $j$ th sensor.

\section{Fault Detection and Isolation FDI Strategy}

3.1. Problem Statement. It is important to be able to carry out the fault detection and isolation before that the faults induce a drastic effect on the system performance. Even in the case of system changes, faults should be detected and isolated. The observer-based approach is used to generate residual signals corresponding to the difference between measured and estimated signals. It is straightforward to think that if the system is faulty, the residual signal will be different to zero. However, the resultant residual will be equal to zero in case of an unfaulty system. The residual signal is compared to a fixed threshold; this comparison is followed by a decision block. To handle all possible sensor faults, we use an observer's bank composed of three high gain observers. Each observer uses the information of two sensors to estimate the third state as it is shown in Figure 2.

The three estimated liquid levels $\widehat{y}_{1}, \hat{y}_{2}$, and $\widehat{y}_{3}$ provided by the observers' bank allow to calculate the three residuals as follows:

$$
r_{j}=y_{j}-\widehat{y}_{j}, \quad \text { for } j=1, \ldots, 3 \text {. }
$$

Considering these following notations, Table 1 can be established:

$$
\begin{gathered}
\text { sensor }_{j}= \begin{cases}0, & \text { fault in the } j^{\text {th }} \text { sensor, } \\
1, & \text { no fault in the } j^{\text {th }} \text { sensor, }\end{cases} \\
r_{j}= \begin{cases}\neq 0, & \text { fault in the } j^{\text {th }} \text { sensor, } \\
0, & \text { no fault in the } j^{\text {th }} \text { sensor. }\end{cases}
\end{gathered}
$$

Consequently, the novel output vector used to implement the control law is given by

$$
y_{c}=\left(\begin{array}{c}
y_{c 1} \\
y_{c 2} \\
y_{c 3}
\end{array}\right) ; \quad \text { where } y_{c j}=\overline{F_{j}} \cdot y_{j}+F_{j} \cdot \widehat{y}_{j},
$$

with $y_{j}$ and $\widehat{y}_{j}$ are, respectively, the measured and the estimated output. $F_{j}$ is a binary variable such as

$$
F_{j}= \begin{cases}1, & \text { fault in the } j \text { th sensor, } \\ 0, & \text { no fault in the } j \text { th sensor. }\end{cases}
$$

Once the FDI is performed, the faulty sensor $S_{i}$ is identified and the binary variable $F_{i}$ is set to 1 . As a result, the control output $y_{c i}$ switches from measured to the estimated output provided by the $i^{\text {th }}$ observer (see Figure 3 ).

\subsection{Observer Design}

3.2.1. Basic Concepts. Consider nonlinear systems of the form:

$$
\left\{\begin{array}{l}
\dot{x}=f(x, u), \\
y=p(x),
\end{array}\right.
$$

where the vectors $x$ and $u$ are, respectively, the state and control defined on the subsets $M$ and $U$ and the vectorvalued functions $f($.$) and p($.) are sufficiently differentiable with respect to their arguments.

Firstly, assume $y$ is a single output. Suppose an injective map $\zeta=q(x)$ exists, which has a continuous inverse and brings system (12) into the bitriangular form:

$$
\begin{aligned}
{\left[\begin{array}{c}
\dot{\zeta} \\
\vdots \\
\dot{\zeta}_{k-1} \\
\dot{\zeta}_{k}
\end{array}\right] } & =\left[\begin{array}{c}
\zeta_{2}+\psi\left(\zeta_{1}, u\right) \\
\vdots \\
\zeta_{k}+\psi_{k-1}\left(\zeta_{1}, \ldots, \zeta_{k-1}, u\right) \\
\psi_{k}\left(\zeta_{1}, \ldots, \zeta_{k}, u\right)
\end{array}\right], \\
y & =\zeta_{1} .
\end{aligned}
$$

Denote the system in the $\zeta$-coordinate as 


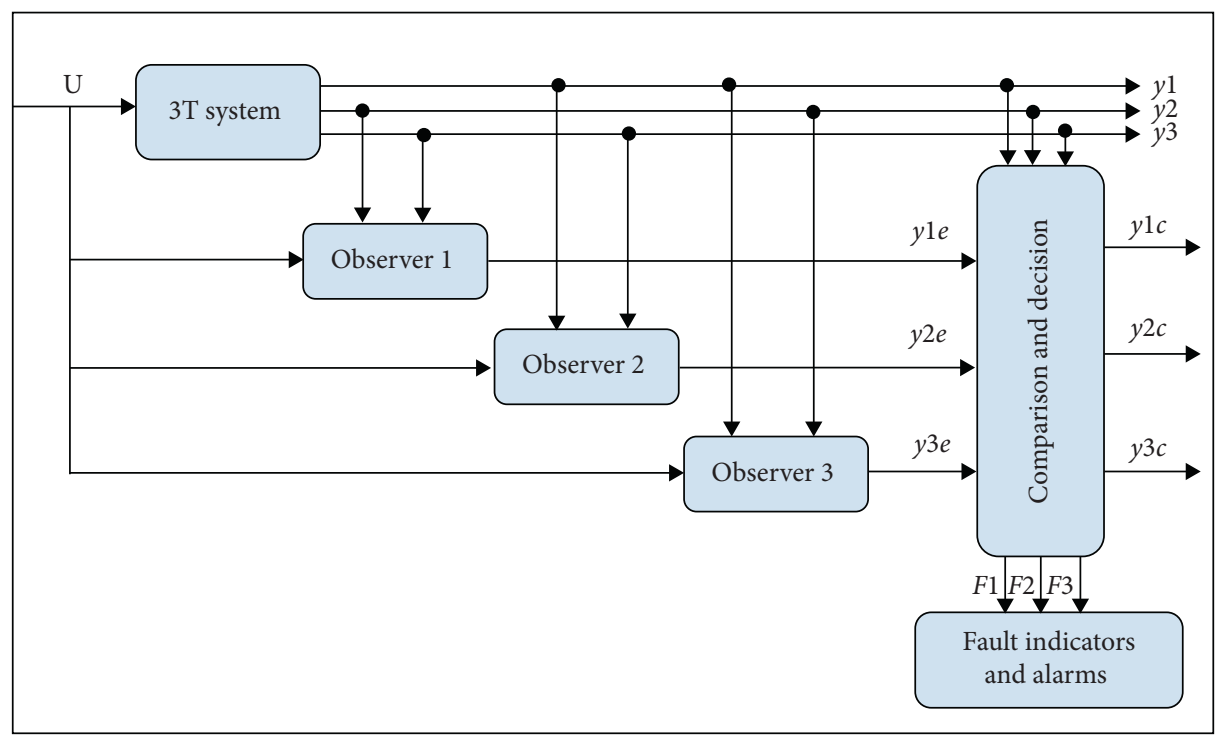

FIgURE 2: Synoptic scheme of FDI applied to 3-tank system.

TABLE 1: Faults signature for the three residuals.

\begin{tabular}{lcccccccc}
\hline Sensor 1 & Sensor 2 & Sensor 3 & $r_{1}$ & $r_{2}$ & $r_{3}$ & $\mathrm{~F}_{1}$ & $\mathrm{~F}_{2}$ & $\mathrm{~F}_{3}$ \\
\hline 1 & 1 & 1 & 0 & 0 & 0 & 0 & 0 & 0 \\
0 & 1 & 1 & $\neq 0$ & 0 & 0 & 1 & 0 & 0 \\
1 & 0 & 1 & 0 & $\neq 0$ & 0 & 0 & 1 & 0 \\
1 & 1 & 0 & 0 & 0 & $\neq 0$ & 0 & 0 & 1 \\
0 & 0 & 1 & $\neq 0$ & $\neq 0$ & 0 & 1 & 1 & 0 \\
1 & 0 & 0 & 0 & $\neq 0$ & $\neq 0$ & 0 & 1 & 1 \\
0 & 1 & 0 & $\neq 0$ & 0 & $\neq 0$ & 1 & 0 & 1 \\
0 & 0 & 0 & $\neq 0$ & $\neq 0$ & $\neq 0$ & 1 & 1 & 1
\end{tabular}

$$
\dot{\zeta}=A \zeta+\psi(\zeta, u), \quad y=C \zeta
$$

with

$$
\begin{aligned}
A & =\left[\begin{array}{ccccc}
0 & 1 & & & \\
& \ddots & \ddots & & \\
& & \ddots & \ddots & \\
& & & \ddots & 1 \\
& & & & 0
\end{array}\right], \\
C & =\left[\begin{array}{cccc}
1 & 0 & \cdots & 0
\end{array}\right], \\
\psi(.) & =\left[\begin{array}{c}
\psi\left(\zeta_{1}, u\right) \\
\vdots \\
\psi_{k-1}\left(\zeta_{1}, \ldots, \zeta_{k-1}, u\right) \\
\psi_{k}\left(\zeta_{1}, \ldots, \zeta_{k}, u\right)
\end{array}\right] .
\end{aligned}
$$

Assume in the coordinate change $\operatorname{dim}[\zeta]=\operatorname{dim}[x]$ and the Jacobian $(\partial q(x) / \partial x)$ is nonsingular. According to [30], for (12) an asymptotic observer is

$$
\dot{\hat{x}}=f(\hat{x}, u)+\left(\left.\frac{\partial q(x)}{\partial x}\right|_{x=\hat{x}}\right)^{-1} L_{\theta}\left(y_{i}-p(\hat{x})\right),
$$

where $L_{\theta}=\left[\begin{array}{c}\theta L_{1} \\ \vdots \\ \theta^{k} L_{k}\end{array}\right]$ and $\theta>0$ is a scalar; the size of $L_{i}$ is equal to the dimension of $\zeta_{i}$.
In a practical design, $L$ is firstly chosen such that $A-L C$ is stable. Then, an arbitrary $\theta \geq 1$ may be chosen. A large value of $\theta$ involved relatively a fast convergence in the estimation error, but, at the same time, it can induce an amplification of the noise measurement.

3.2.2. HGO Design for the 3TS Model. The nonlinear model (4) of the three-tank system can be written as follows:

$$
\dot{h}=f(h)+g Q
$$

where

$$
\begin{gathered}
h=\left[\begin{array}{l}
h_{1} \\
h_{2} \\
h_{3}
\end{array}\right], \\
Q=\left[\begin{array}{l}
Q_{1} \\
Q_{2}
\end{array}\right], \\
g=\left[\begin{array}{ll}
\frac{1}{S} & 0 \\
0 & 0 \\
0 & \frac{1}{S}
\end{array}\right],
\end{gathered}
$$$$
f(h)=\left[\begin{array}{c}
-a_{1} \operatorname{sgn}\left(h_{1}-h_{2}\right) \sqrt{\left|h_{1}-h_{2}\right|} \\
a_{1} \operatorname{sgn}\left(h_{1}-h_{2}\right) \sqrt{\left|h_{1}-h_{2}\right|}-a_{2} \operatorname{sgn}\left(h_{2}-h_{3}\right) \sqrt{\left|h_{2}-h_{3}\right|} \\
a_{2} \operatorname{sgn}\left(h_{2}-h_{3}\right) \sqrt{\left|h_{2}-h_{3}\right|}-a_{3} \sqrt{h_{3}}
\end{array}\right] .
$$ 


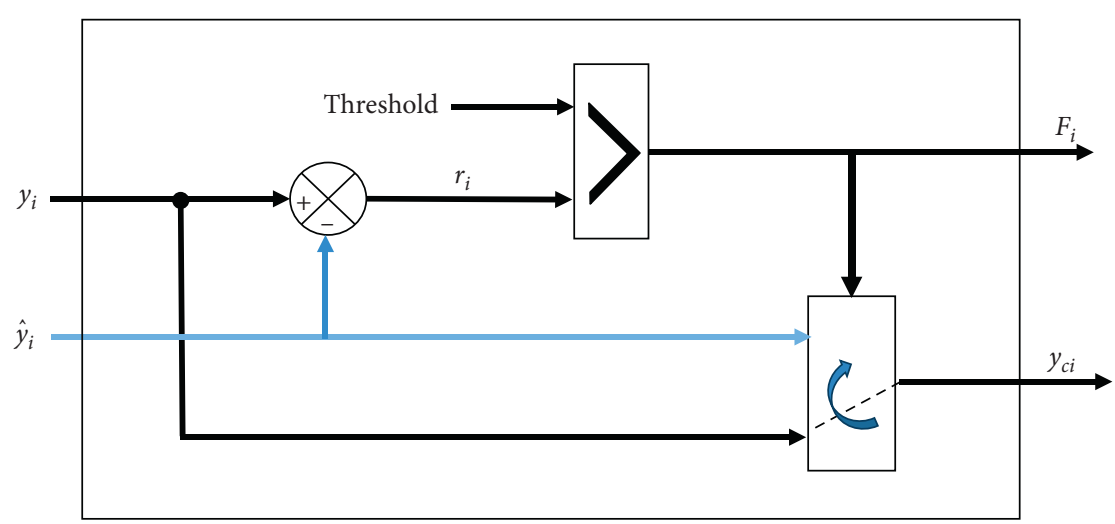

FIGURE 3: Internal diagram of comparison and decision unit.

Typical values of the $3 \mathrm{~T}$ system are given in Table 2 . These values are later used in observer and controller implementation.

(1) Observer Form with a Single Output Measurement. If only a single measurement is available during operating, according to (16), the observer for the 3TS model takes the form

$$
\dot{\hat{h}}=f(\widehat{h})+g(Q)+\left(\left.\frac{\partial q_{i j}(h)}{\partial h}\right|_{h=\hat{h}}\right)^{-1} L_{\theta}\left(y_{i}-\widehat{h}_{i}\right),
$$

where

$$
\begin{aligned}
L_{\theta} & =\left[\begin{array}{c}
\theta l_{1} \\
\theta^{2} l_{2} \\
\theta^{3} l_{3}
\end{array}\right], \quad \theta \geq 1, \\
L & =\left[\begin{array}{l}
L_{1} \\
L_{2} \\
L_{3}
\end{array}\right],
\end{aligned}
$$

ensuring that the matrix $A-L C$ is Hurwitz:

$$
\begin{aligned}
& A=\left[\begin{array}{lll}
0 & 1 & 0 \\
0 & 0 & 1 \\
0 & 0 & 0
\end{array}\right], \\
& C=\left[\begin{array}{lll}
1 & 0 & 0
\end{array}\right] .
\end{aligned}
$$

For instance, suppose only $y_{1}=h_{1}$ is available. Then, $\zeta=$ $q_{1}(h)$ with

$$
\begin{aligned}
q_{1}(h) & =\left[\begin{array}{c}
h_{1} \\
L_{f} h_{1} \\
L_{f}^{2} h_{1}
\end{array}\right] \\
& =\left[\begin{array}{c}
h_{1} \\
-a_{1} \operatorname{sign}\left(h_{1}-h_{2}\right) \sqrt{\left|h_{1}-h_{2}\right|} \\
a_{1}^{2} \operatorname{sign}\left(h_{1}-h_{2}\right) \sqrt{\left|h_{1}-h_{2}\right|}-\frac{a_{1} a_{2} \operatorname{sign}\left(h_{2}-h_{3}\right) \sqrt{\left|h_{2}-h_{3}\right|}}{2 \sqrt{h_{1}-h_{2}}}
\end{array}\right],
\end{aligned}
$$

TABLE 2: Physical parameters of the three-tank system.

\begin{tabular}{lc}
\hline Tank cross section areas & $S_{1}=S_{2}=S_{3}=S=0.01 \mathrm{~m}^{2}$ \\
Pipe cross section areas & $S_{\mathrm{N}}=0.0000786 \mathrm{~m}^{2}$ \\
Coefficients & $a_{1}=0.1 ; a_{2}=0.086 ; a_{3}=0.099$ \\
Maximum in-flow rate & $Q_{\max }=6.66 .10^{-5} \mathrm{~m}^{3} / \mathrm{s}$ \\
\hline
\end{tabular}

where $L_{f}^{j} h(x)$ is the $\mathrm{j}^{\text {th }}$ Lie derivative of the function $h$ by $f$; for example, $h(x)$ and $f(x)$ are differentiable functions of $x$ up to the order $n$.

So, we have

$$
\frac{\partial q_{1}}{\partial h}=\left[\begin{array}{ccc}
1 & 0 & 0 \\
-a & a & 0 \\
b & -c-b & c
\end{array}\right]
$$

with

$$
\begin{aligned}
& a=\frac{a_{1}}{2 \sqrt{\left|h_{1}-h_{2}\right|}} ; \\
& b=\frac{a_{1} a_{2} \operatorname{sgn}\left(h_{2}-h_{3}\right) \sqrt{\left|h_{1}-h_{2}\right|}}{4 \sqrt{\left|h_{1}-h_{2}\right|\left|h_{1}-h_{2}\right|}} ; \\
& c=\frac{a_{1} a_{2}}{4 \sqrt{\left|h_{1}-h_{2}\right| \cdot\left|h_{2}-h_{3}\right|}},
\end{aligned}
$$

and hence

$$
\left(\frac{\partial q_{1}}{\partial h}\right)^{-1}=\left(\begin{array}{ccc}
1 & 0 & 0 \\
1 & \frac{1}{a} & 0 \\
1 & \frac{1}{a}+\frac{b}{c a} & \frac{1}{c}
\end{array}\right)
$$

(2) Observer Form with Both State Measurements. To detect and isolate several sensor faults in the whole operating area, we use in FDI block a bank of nonlinear observers. According to [30] and using the assumption cited in [31] if 
two measurements $y i$ and $y j$ are available, the $\mathrm{HGO}$ is given by

$$
\dot{\hat{h}}=f(\widehat{h})+g(Q)+\left(\left.\frac{\partial q_{i j}(h)}{\partial h}\right|_{h=\hat{h}}\right)^{-1} L_{\theta}\left(y_{i, j}-\widehat{h}_{i, j}\right),
$$

where

$$
\begin{aligned}
L_{\theta} & =\left[\begin{array}{c}
L_{1} \theta \\
L_{2} \theta^{2}
\end{array}\right], \quad \theta \geq 1, \\
L & =\left[\begin{array}{c}
L_{1} \\
L_{2}
\end{array}\right], \\
L & =\left[\begin{array}{c}
L_{1} \\
L_{2}
\end{array}\right],
\end{aligned}
$$

where $L 1$ and $L 2$ are, respectively, $2 \times 2$ and $1 \times 2$ constant matrix, which can be easily determined such that $A-L C$ has merely stable eigenvalues (26).

(i) b.1-Observer 1. Suppose $y_{2}=h_{2}$ and $y_{3}=h_{3}$ are the two available measurements. Let $\varepsilon_{1}=\left[\begin{array}{l}h_{2} \\ h_{3}\end{array}\right]$ and $\varepsilon_{2}=L_{f} h_{2}$ is the Lie derivative of $h 2$ by $f:$

$$
\begin{aligned}
\mathcal{E} & =\left[\begin{array}{l}
\varepsilon_{1} \\
\varepsilon_{2}
\end{array}\right]=q_{23}(h)=\left[\begin{array}{c}
h_{2} \\
h_{3} \\
L_{f} h_{2}
\end{array}\right], \\
y_{2,3}-\widehat{h}_{2,3} & =\left[\begin{array}{l}
y_{2} \\
y_{3}
\end{array}\right]-\left[\begin{array}{c}
\widehat{h}_{2} \\
\widehat{h}_{3}
\end{array}\right], \\
\left(\frac{\partial q_{23}}{\partial h}\right)^{-1} & =\left[\begin{array}{ccc}
1+\frac{b}{a} & -\frac{b}{a} & \frac{1}{a} \\
1 & 0 & 0 \\
0 & 1 & 0
\end{array}\right],
\end{aligned}
$$

(ii) with

$$
\begin{aligned}
& a=\frac{a_{1}}{2 \sqrt{\left|h_{1}-h_{2}\right|}} ; \\
& b=\frac{a_{2}}{2 \sqrt{\left|h_{2}-h_{3}\right|}} ; \\
& L=\left[\begin{array}{l}
L_{1} \\
L_{2}
\end{array}\right],
\end{aligned}
$$

(iii) where the dimensions of $L 1$ and $L 2$ are, respectively, $2 \times 2$ and $1 \times 2$ constant matrix, which can be easily determined such that $A-L C$ has merely stable eigenvalues. In this case, we have

$$
\begin{aligned}
A & =\left[\begin{array}{lll}
0 & 0 & 1 \\
0 & 0 & 0 \\
0 & 0 & 0
\end{array}\right] ; \\
C & =\left[\begin{array}{lll}
1 & 0 & 0 \\
0 & 1 & 0
\end{array}\right] ; \\
L_{\theta} & =\left[\begin{array}{c}
L_{1} \theta \\
L_{2} \theta^{2}
\end{array}\right] .
\end{aligned}
$$

(iv) b.2-Observer 2. Suppose $y 1=h 1$ and $y 3=h 3$ are the two available measurements; $\varepsilon_{1}^{\prime}=\left[\begin{array}{l}h_{1} \\ h_{3}\end{array}\right]$; and $\varepsilon_{2}^{\prime}=$ $L_{f} h_{1}$ is the Lie derivative of $h 1$ by $f$ :

$$
\begin{aligned}
\varepsilon^{\prime} & =\left[\begin{array}{c}
\varepsilon_{1}^{\prime} \\
\varepsilon_{2}^{\prime}
\end{array}\right]=q_{21}(h)=\left[\begin{array}{c}
h_{1} \\
h_{3} \\
L_{f} h_{1}
\end{array}\right], \\
y_{2,1}-\widehat{h}_{2,1} & =\left[\begin{array}{l}
y_{1} \\
y_{3}
\end{array}\right]-\left[\begin{array}{l}
\widehat{h}_{1} \\
\widehat{h}_{3}
\end{array}\right],
\end{aligned}
$$

$$
\left(\frac{\partial q_{13}}{\partial h}\right)^{-1}=\left[\begin{array}{ccc}
1 & 1 & 0 \\
0 & 0 & 1 \\
0 & \frac{1}{a} & 0
\end{array}\right] \text {, }
$$

(v) with $a$ and $b$ as given below, and $L \theta$ is the same as given in observer 1 .

(vi) b.3-Observer 3. Suppose $y_{1}=h_{1}$ and $y_{2}=h_{2}$ are available. Let $\varepsilon_{1}^{\prime \prime}=\left[\begin{array}{l}h_{2} \\ h_{1}\end{array}\right]$ and $\varepsilon_{2}^{\prime \prime}=L_{f} h_{2}$ is the Lie derivative of $h 2$ by $f$ :

$$
\begin{aligned}
& \varepsilon^{\prime \prime}=\left[\begin{array}{c}
\prime \prime \\
\varepsilon_{1} \\
\prime \prime \\
\varepsilon_{2}
\end{array}\right]=q_{21}(h)=\left[\begin{array}{c}
h_{2} \\
h_{1} \\
L_{f} h_{2}
\end{array}\right], \\
& y_{2,1}-\widehat{h}_{2,1}=\left[\begin{array}{l}
y_{2} \\
y_{1}
\end{array}\right]-\left[\begin{array}{l}
\widehat{h}_{2} \\
\widehat{h}_{1}
\end{array}\right], \\
&\left(\frac{\partial q_{21}}{\partial h}\right)^{-1}=\left[\begin{array}{ccc}
0 & 1 & 0 \\
1 & 0 & 0 \\
1+\frac{a}{b} & -\frac{a}{b} & \frac{1}{b}
\end{array}\right],
\end{aligned}
$$

Where $L \theta$ is the same as given in observer 1 .

The proof of convergence of this observer is detailed in [31]. 


\section{FTC Design}

The main objective is to establish a closed-loop regulation to track two reference liquid levels. For this reason, two PI controllers are installed. Each one controls one liquid level. In safety mode, these controllers can successfully accomplish this task. However, in the case of a faulty sensor, nominal performances are affected in the best case, and it can lead to instability in the worst case. To avoid such behavior, we should use the FTC that allows reconfiguring the controller when a fault occurs. As is shown in Figure 4, the role of the FTC unit can be divided into two main tasks: the first one is the FDI detailed in Section 3 and the second one concerns the control law reconfiguration which performs the design parameters adjustment. In the case of faulty sensor $\mathrm{Si}$, the binary variable $\mathrm{Fi}$ is set to 1. As a result, the control output yci switches from measured to the estimated output provided by the $i$ th observer. To reduce the sensor fault effect and to maintain the closedloop performance, the control output yci is used for the feedback and then compared with the input reference.

The sensor fault accommodation allows reducing the fault effect, and so the system still operates in the degraded mode. To alleviate this degradation, we suggest adjusting the parameters of the PI controller $\left(k_{p}, k_{i}\right)$, from the normal mode parameters $\left(k_{p i n}, k_{i i n}\right)$ to faulty mode parameters $\left(k_{p f}\right.$, $k_{i f}$ ) using a switcher block.

\section{FTC Application}

To prove the validity of the FTC strategy proposed in paragraphs 3 and 4, we apply it to the hydrographic system described in the second section. The main aim is to accomplish a closed-loop regulation of two levels $h_{1}$ and $h_{3}$. As is shown in Figure 5, the test setup is composed by the following:

(i) Three liquid tanks with 1-meter height and one evacuation reservoir.

(ii) Two $36 \mathrm{~W}$ water-pumps $(12 \mathrm{~V} / 3 \mathrm{~A})$ with a $4 \mathrm{Lpm}$ liquid flow.

(iii) Three piezoresistive transducers MPX-5010 with sensitivity equal to $450 \mathrm{mV} / \mathrm{kPa}$; everyone equips each tank. These transducers provide accurate analog output signals that are proportional to the pressure variation due to the liquid injection. The accepted range of pressure is from 0 to $10 \mathrm{kPa}$ and the output signal is between 0.2 and $4.7 \mathrm{v}$. The main feature of this sensor is the possibility to connect it directly to a microcontroller without using a conditioning card.

(iv) An STM32Fio card is used as an I/O interface to establish a connection, via USB port, between the control desk using MATLAB/Simulink environment and the I/O peripheries (the three piezoresistive differential pressure sensors as input and the motopump drive board as output).

(v) A control desk with MATLAB/Simulink environment. (vi) Power supply of two variable voltage sources $30 \mathrm{~V} /$ $4 \mathrm{~A}$.

The control law is implemented in real time using a sampling period of $0.1 \mathrm{~s}$.

The experimental setup is in the laboratory "Study of Industrial Systems and Renewable Energies" "ESIER" at the National Engineers School of Monastir, Tunisia.

5.1. Fault Free Case. In a faulty free case, the PI controllers successfully ensure this task since these outputs track well the desired trajectory as is shown in Figure 6.

To ensure good tracking of the level references $h_{1}$ and $h_{3}$, we used two PI controllers, one for each level with identical parameters $K_{p n}=20$ and $K_{i n}=15$.

\subsection{Faulty Cases}

\subsubsection{Fault Scenarios}

(i) At $t 1=300 \mathrm{~s}$, a constant offset of $-8 \mathrm{~cm}$ is added to liquid level $h_{1}\left(\beta 1=1, y_{10}=-8 \mathrm{~cm}\right)$.

(ii) At $t 2=500 \mathrm{~s}$, a constant offset of $-6 \mathrm{~cm}$ is added to liquid level $h_{3}\left(\beta 3=1, y_{30}=-6 \mathrm{~cm}\right)$.

5.2.2. Sensor Fault Effect without FTC. The consequence of the fault scenario in the feedback performance is illustrated in Figure 7. From the instant $t 1=300 \mathrm{~s}$, the measured level $h_{1}$ has a bias of $-8 \mathrm{~cm}$ compared to its real value; that is why the control law tries to cancel the static error created by the faulty measurement which appears clearly in the sudden magnitude change of $u_{1}$. Consequently, the real output is different from the reference and it is equal to the value of reference plus the bias value $(37.5+8=43.5 \mathrm{~cm})$. Since $t=450 \mathrm{~s}$, the control law $u_{1}$ has been almost constant and greater than the nominal value in the fault-free case. Similar to level 1 fault consequence, Figure 8 shows the effect of the fault in sensor 3 which arises at a real level different from the reference, and after $t=720 \mathrm{~s}$, a control law $u_{2}$ which has almost a constant value greater than the nominal one.

5.2.3. FTC without Controller Adjustment. The application of the previous FDI method to the same fault scenario sited in subsection 5.2.1 requires the use of observers' bank in order to generate residual and to identify the faulty sensor. After a transient time, this residual is compared to a fixed threshold which allows setting the binary variable $F i$ to 1 or 0 .

(i) At $t=t 1=300 \mathrm{~s}$, a bias of $-8 \mathrm{~cm}$ is added to the liquid level 1; this sudden change induces a rocking of the control output $y_{\mathrm{c} 1}$ from the measured $y_{1}$ to the estimated $\widehat{y}_{1}$ generated by the observer 1 (see Figures 2 and 3 ).

(ii) At $t=t 2=500 \mathrm{~s}$, similar to level 1 , a bias of $-6 \mathrm{~cm}$ is added to the third level and, consequently, the feedback is ensured by the estimated output $\widehat{y}_{3}$. 


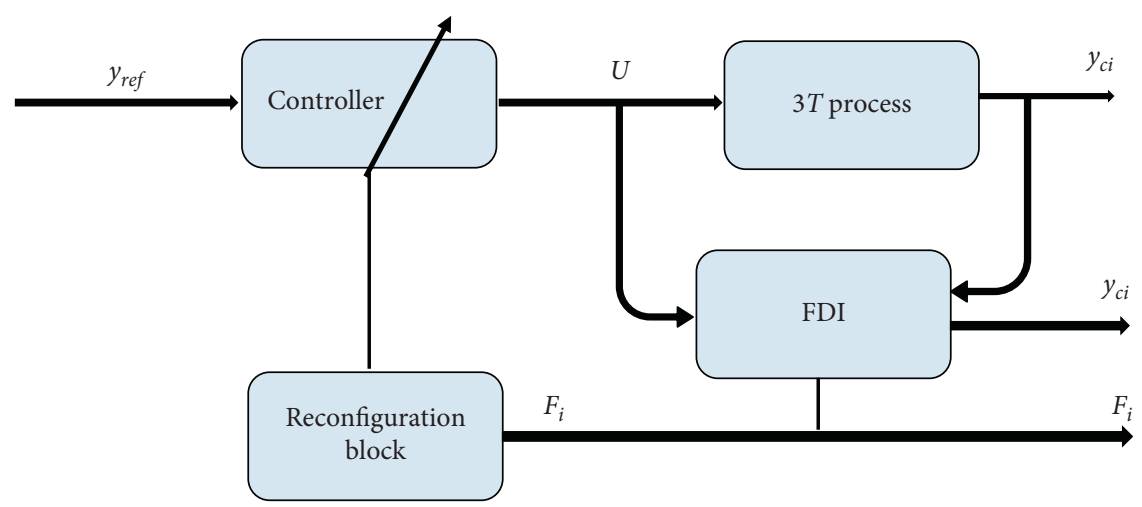

FIgURE 4: Synoptic scheme of an FTC including FDI module.

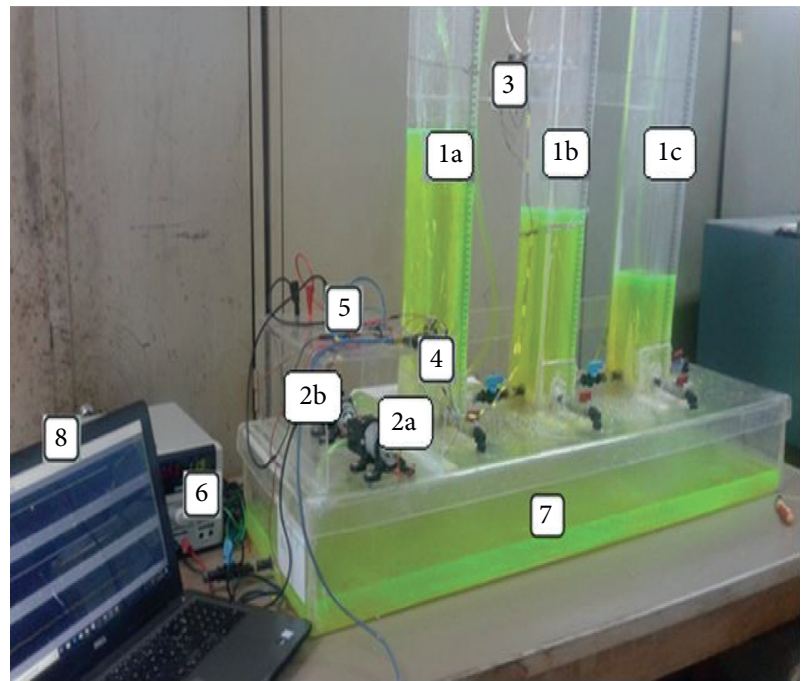

FIgURE 5: Experimental plant: (1a, 1b, 1c) 3 cubic tanks; (2a, 2b) 2 DC motopumps; (3) liquid level sensors; (4) Fio std STM32 board; (5) motor driver board; (6) power supply; (7) basin; and (8) control desk.

In a practical design, $L$ is firstly chosen such that $A$-LC is Hurwitz. Then, an arbitrary $\theta \geq 1$ may be chosen. Normally, a large value of $\theta$ allows a fast estimation error convergence. But, in the same way, it can generate excessive peaks during the transient, beside inducing an amplification of the noise measurement in the state estimation. To achieve a compromise, we have chosen the observers' parameters as $\theta_{1}=\theta_{2}=\theta_{3}=2$.

As is shown in Figures 9 and 10, levels 1 and 3 try to suitably track the liquid reference trajectories. But, since the comparison of the input reference is done with an estimated value of the real measurement, large oscillations appear after the fault occurrence.

5.2.4. FTC with Controller Adjustment. To improve the performance of the closed-loop sited lastly, we suggest the adjustment of the controller parameters from the nominal one $\left(K_{p n}=20, K_{i} n=15\right)$ to the faulty one $\left(K_{p f}=70\right.$, $\left.K_{i f}=50\right)$ using a switcher block in which the switching
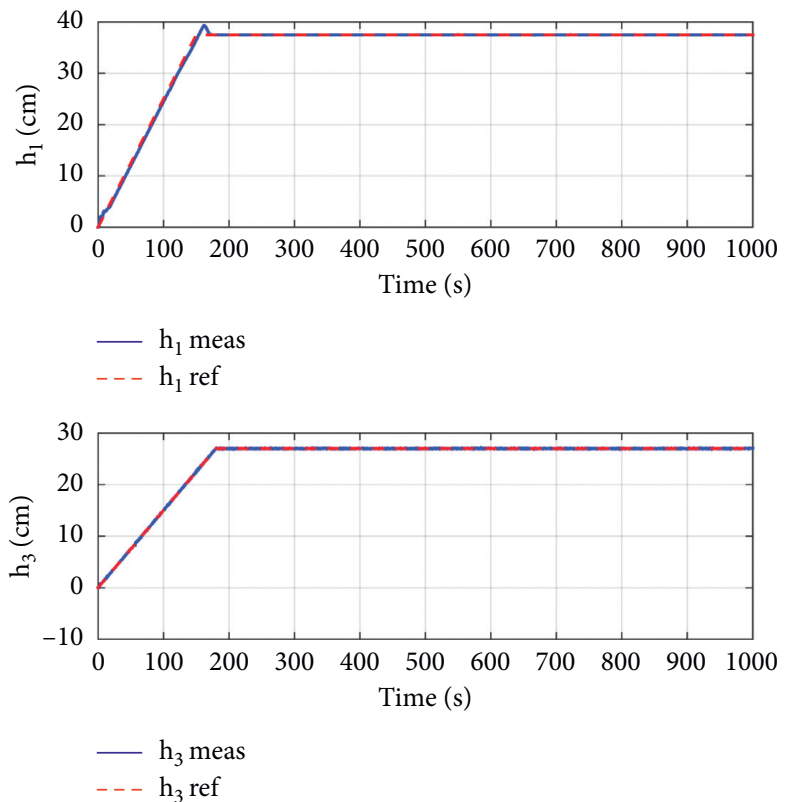

FIGURE 6: Tracking performances of liquid levels 1 and 3 in the fault-free case.

condition is the error between the estimated and the measured signals. As is shown in Figure 11, in a healthy case, the error is less than the threshold; then $K_{p}=K_{p n}$, after fault occurrence, the error is greater than the threshold and $K_{p}$ switch to $K_{p f}$.

The result of this adjustment is shown in Figures 12 and 13. Compared to Figures 9 and 10, the quality of regulation is improved.

5.2.5. Result Discussion. The proposed active fault-tolerant control ensures typically quite performances for the closedloop system. Indeed, after sensor fault accommodation a controller reconfiguration is performed to improve the tracking performances. The tracking errors $e_{1}=y_{1}-y_{1 \text { ref }}$ and $e_{3}=y_{3}-y_{3 \text { ref }}$ are depicted, respectively, in Figures 14 and 15 for three cases: fault-free case (a), FTC without control law adjustment (b), and FTC with control law adjustment (c). 


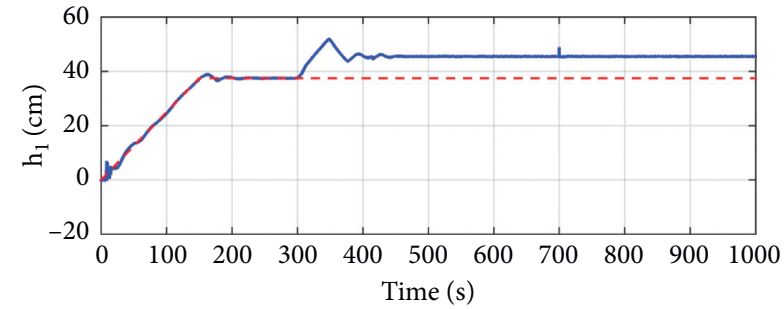

- $\mathrm{h}_{1}$ without $\mathrm{ftc}$

$---h_{1}$ ref

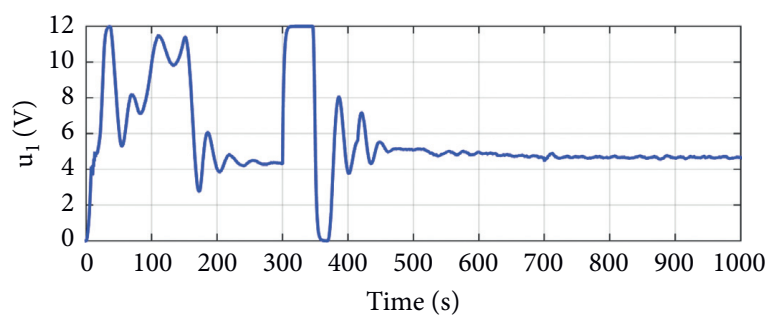

FIGURE 7: Dynamic response of level 1 without FTC and its control law $u_{1}$.
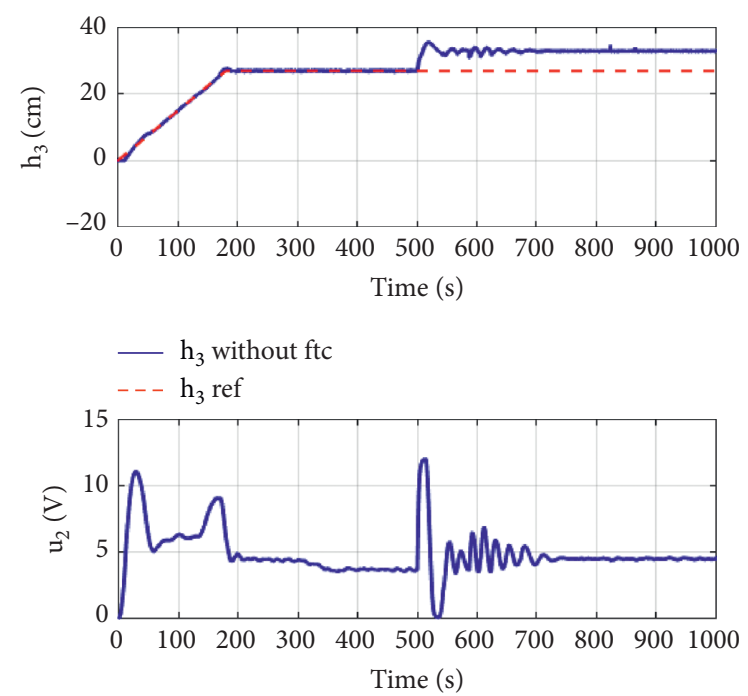

Figure 8: Dynamic response of level 3 without FTC and its control law $u_{2}$.

To evaluate the control performance, we use the mean square error MSE criterion defined by

$$
\operatorname{MSE}=\frac{1}{n} \sum_{k=1}^{n}\left(y_{\text {ref }}(k)-y(k)\right)^{2},
$$

where $n$ is the number of measurements, $y_{\text {ref }}(k)$ is the desired output, and $y(k)$ is the system output. The computation of the MSE for outputs $y_{1}$ and $y_{3}$ in cases $a, b$, and $c$ is illustrated by Table 3, when $n=10000$.

As seen in Table 3, the MSE values arising from the proposed approach are a little bigger than the fault-free case, but it is still widely smaller than the case of FTC without
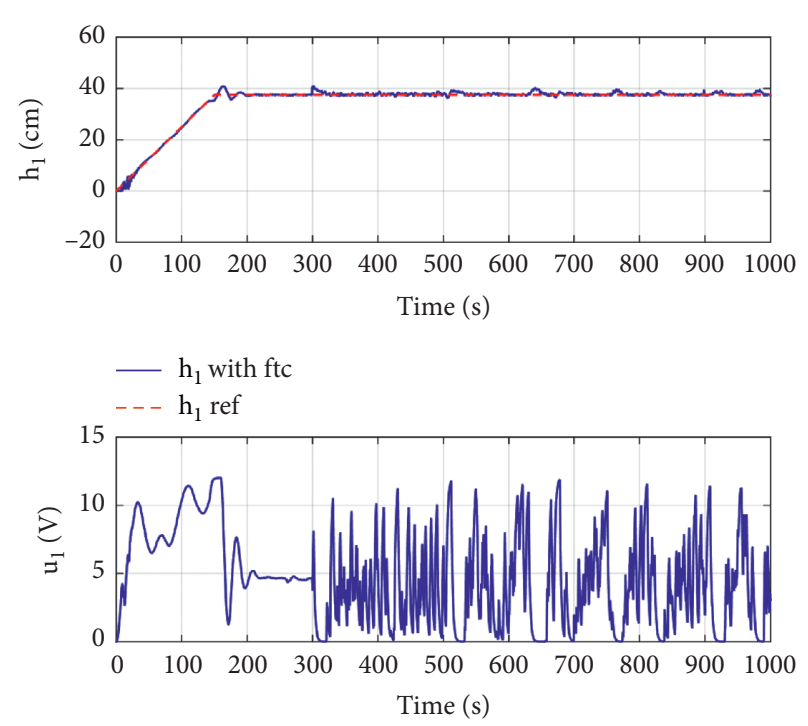

FIGURE 9: Tracking performance of level 1 with FTC and its control law $u_{1}$.

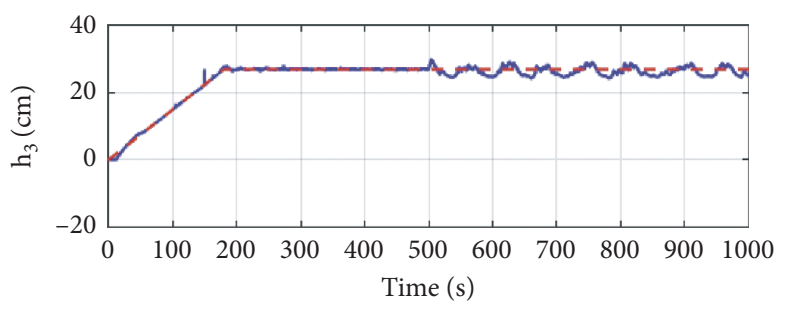

- $\mathrm{h}_{3}$ with $\mathrm{ftc}$

$--\mathrm{h}_{3}$ ref

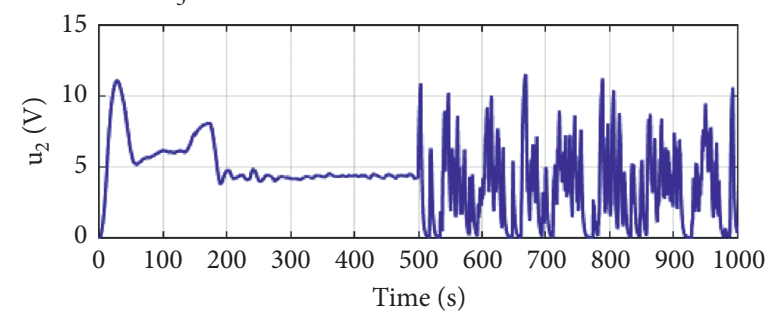

FIgURE 10: Tracking performance of level 3 with FTC and its control law $u_{2}$.

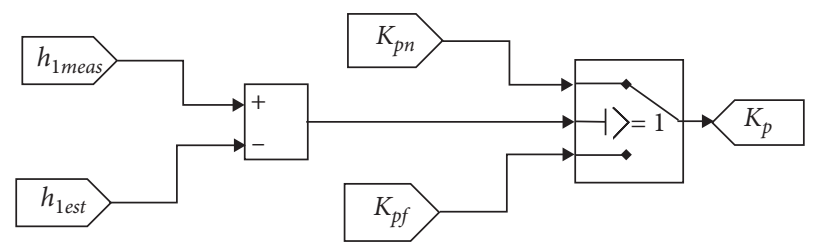

FIgURE 11: Schematic diagram of the controller parameters switching.

control law parameters adjustment. This also is confirmed by the dynamic behavior of the output levels y1 and y3 after sensor fault occurrence. 

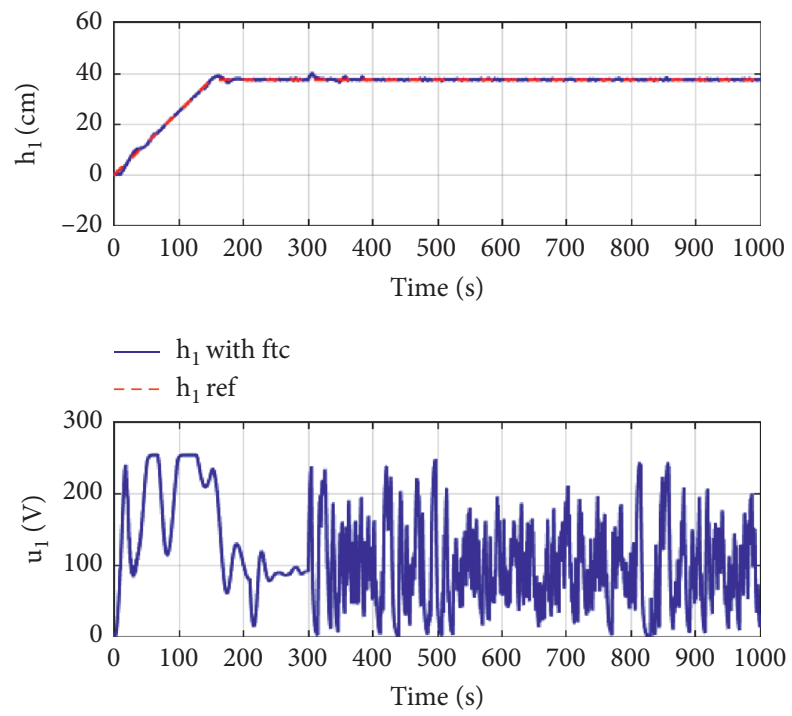

FIgURE 12: Dynamic response of level 1 with FTC and its adjusted control law $u_{1}$.
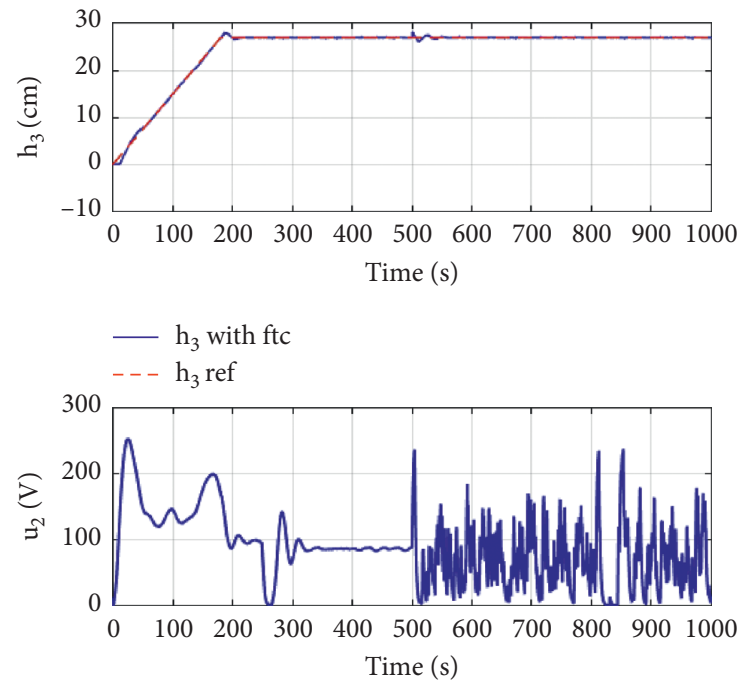

FIgURE 13: Dynamic response of level 3 with FTC and its adjusted control law $u_{2}$.

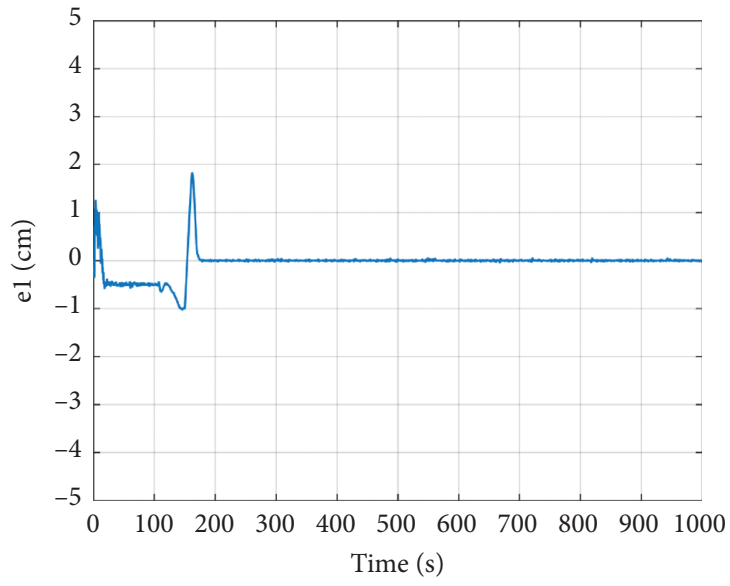

(a)

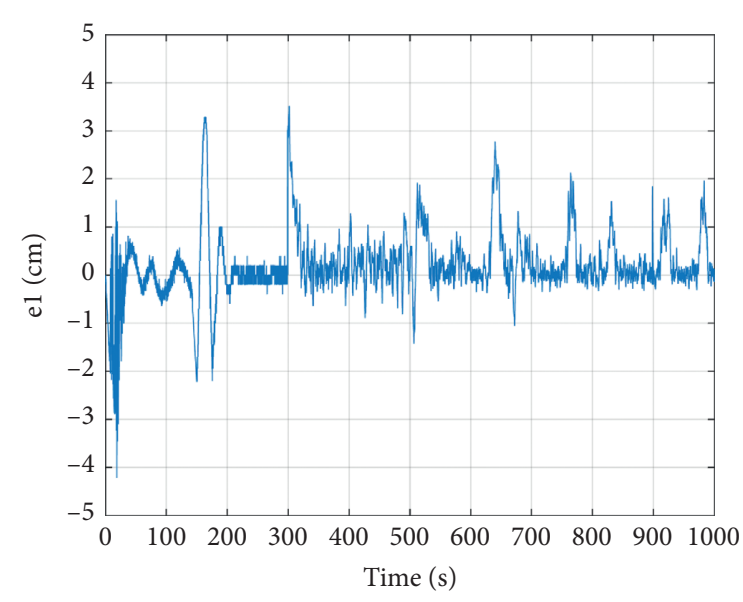

(b)

FIgURE 14: Continued. 


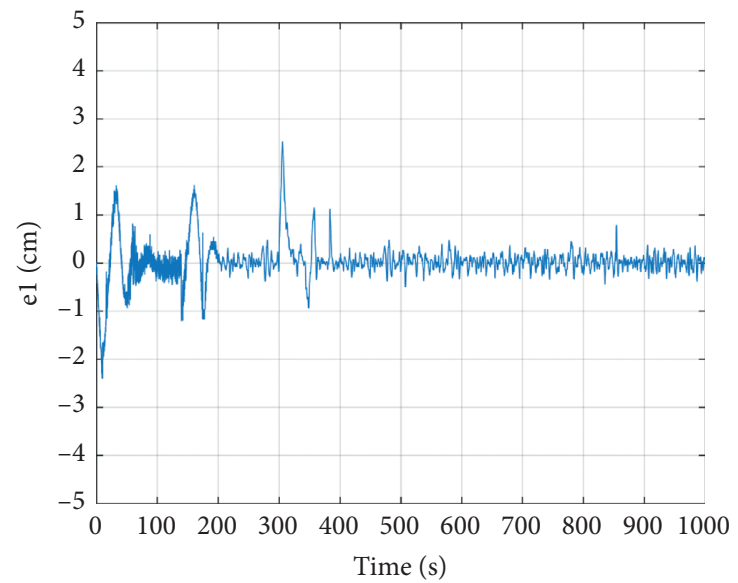

(c)

Figure 14: Tracking error el.

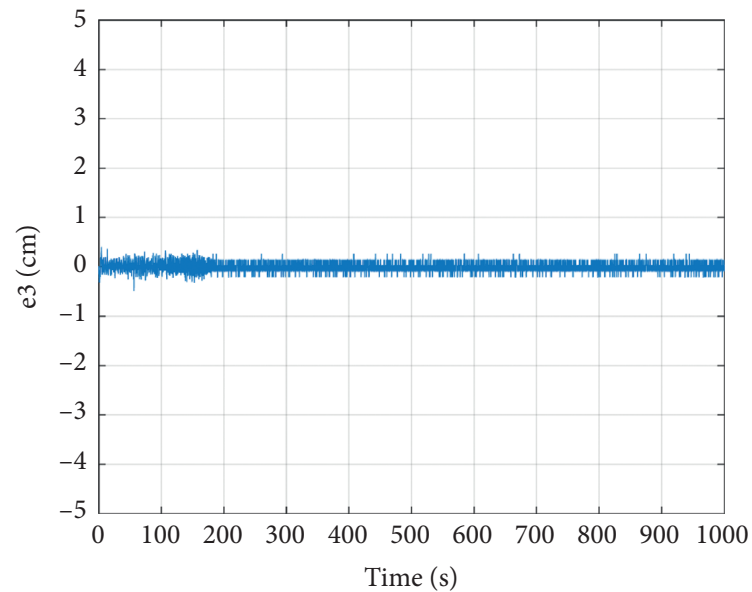

(a)

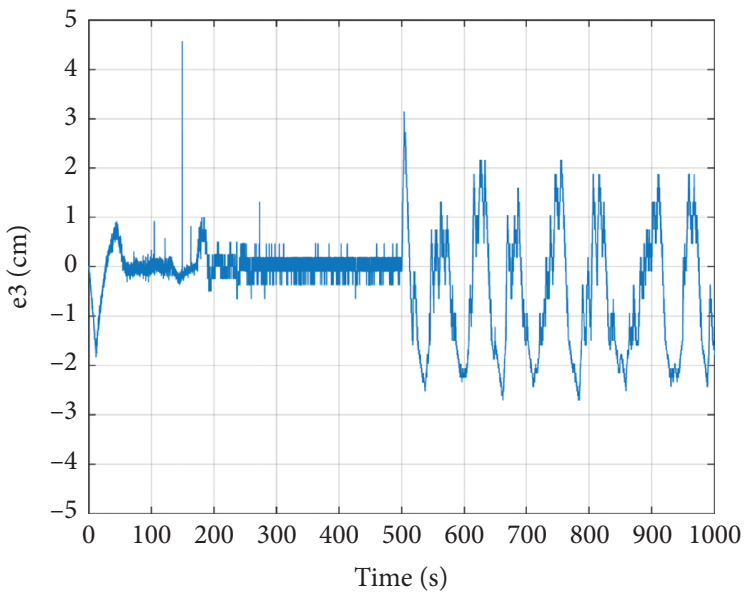

(b)

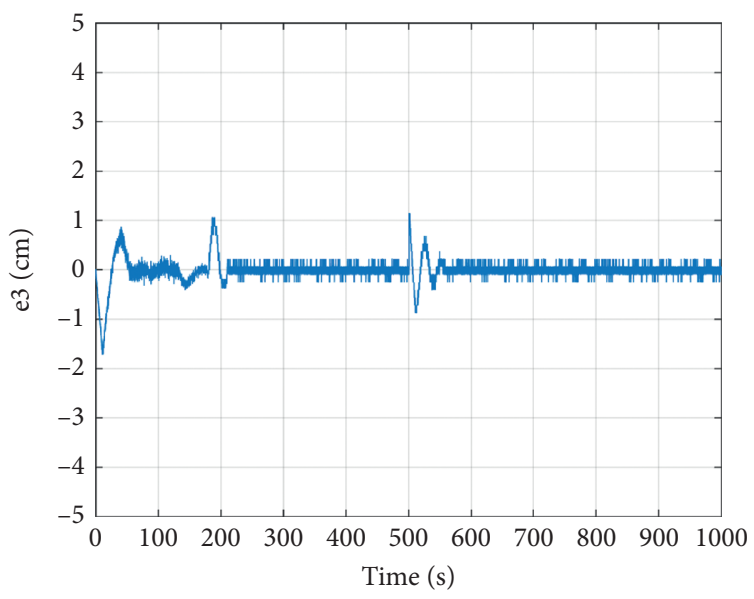

(c)

Figure 15: Tracking error e3.

TABle 3: Performance tracking comparison.

Fault-free case

FTC without parameters adjustment 


\section{Conclusion}

In this study, a bank of high gain observers has been considered for FDI application in a 3TS. After sensor detection and isolation, fault accommodation is applied to ensure the reference tracking aim. For preserving the same performance as the safe mode, a controller parameters adjustment is also proposed. Experimental results are given for the validation of our approach.

This work can be extended to reach simultaneous sensor and actuator faults by using unknown input observers. The performance of the controller feedback can be improved using a filtered high gain observer, which ensures a good state estimation, even in the case of noisy measurements.

\section{Data Availability}

The data used to support the findings of this study are available from the corresponding author upon request.

\section{Conflicts of Interest}

The authors declare that they have no conflicts of interest.

\section{References}

[1] R. J. Patton, "Fault tolerant control": the 1997 situation survey," in Proceedings of the IFAC SAFEPROCESS'97, vol. 2, pp. 1033-1055, Hull, UK, August 1997.

[2] R. Isermann, "Model-based fault detection and diagnosis - status and applications," Annual Review of Control, vol. 29, pp. 71-85, 2005.

[3] M. Kordestani, M. Saif, M. E. Orchard, R. Razavi-Far, and K. Khorasani, "Failure prognosis and applications-A survey of recent literature," IEEE Transactions on Reliability, vol. 99, pp. 1-21, 2019.

[4] J. Chen and R. J. Patton, Robust Model-Based Fault Diagnosis for Dynamic Systems, Kluwer Academic Publisher, London, UK, 1999.

[5] S. Simani, C. Fantuzzi, and R. J. Patton, Model-based Fault Diagnosis in Dynamic Systems Using Identification Techniques, Springer, London, UK, 2003.

[6] W. Yan-Feng, W. Pei-Liang, L. Zu-Xin, and C. Hui-Ying, "Fault-tolerant control for networked control systems with limited information in case of actuator fault," Mathematical Problems in Engineering, vol. 2015, Article ID 785289, 7 pages, 2015.

[7] W. Yan-feng, "Robust fault detection for networked control systems with markov time-delays and data packet loss in both S/C and C/A channels," Mathematical Problems in Engineering, vol. 2019, Article ID 4672862, 11 pages, 2019.

[8] M. Kordestani, K. Salahshoor, A. A. Safavi, and M. Saif, "An adaptive passive fault tolerant control system for a steam turbine using a PCA based inverse neural network control strategy," in Proceedings of the 2018 World Automation Congress (WAC), vol. 40-45, IEEE, Washington, USA, June 2018.

[9] M. Kordestani, A. A. Safavi, N. Sharafi, and M. Saif, "Fault tolerant control of rhine-meuse delta water system: a performance assessment based approach," in Proceedings of the 5th International Conference on Power Generation Systems and Renewable Energy Technologies (PGSRET), Istanbul, Turkey, August 2019.
[10] F. Kimmich, A. Schwarte, and R. Isermann, "Fault detection for mordern diesel engines using signal-and process modelbased methods," Control Enginerering Practice, vol. 13, pp. 189-203, 2005.

[11] L. Min, Z. Zuo, H. Liu, C. Liuand, and B. Zhu, "Adaptive fault tolerant control for trajectory tracking of a quadrotor helicopter," Transactions of the Institute of Measurement and Control, vol. 40, no. 12, pp. 3560-3569, 2018.

[12] F. Boem, A. J. Gallo, D. M. Raimondo, and T. Parisini, "Distributed fault-tolerant control of large-scale systems: an active fault diagnosis approach," IEEE Transactions on Control of Network Systems, vol. 7, no. 1, 2020.

[13] Z. Zhao, Y. Yang, S. X. Ding, and L. Li, "Fault-tolerant control for systems with model uncertainty and multiplicative faults," IEEE Transactions on Systems, Man, and Cybernetics: Systems, vol. 50, no. 2, 2020.

[14] H. Qian, Y. Peng, and M. Cui, "Adaptive observer-based fault-tolerant control design for uncertain systems," Mathematical Problems in Engineering, vol. 2015, Article ID 429361, 16 pages, 2015.

[15] F. Chen and M. Dunnigan, "Comparative study of a slidingmode observer and kalman filters for full state estimation in an induction machine," in IEE Proceedings Electric Power Applicationsvol. 149, pp. 53-64, IET, 2002.

[16] A. Mesbah, A. E. M. Huesman, H. J. M. Kramer, and P. M. J. Van Den Hof, “A comparison of nonlinear observers for output feedback model-based control of seeded batch crystallization processes," Journal of Process Control, vol. 21, no. 4, pp. 652-666, 2011.

[17] E. M. Cimpoesu, B. D. Ciubotaru, and D. Stefanoiu, "fault detection and diagnosis using parameter estimation with recursive least squares," in Proceedings of the 2013 19th International Conference on Control Systems and Computer Science, pp. 18-23, Bucharest, Romania, May 2013.

[18] M. Zhong, T. Xue, Y. Song, S. X. Ding, and E. L. Ding, "Parity space vector machine approach to robust fault detection for linear discrete-time systems," IEEE Transactions on Systems, Man, and Cybernetics: Systems, vol. 51, 2018.

[19] Y. Li and G. Yang, "Fuzzy adaptive output feedback faulttolerant tracking control of a class of uncertain nonlinear systems with nonaffine nonlinear faults," IEEE Transactions on Fuzzy Systems, vol. 24, 2016.

[20] V. Dardinier-Maron, F. Hamelin, and H. Noura, "A faulttolerant control design against major actuator failures: application to a three- tank system," in Proceedings of the 38 th Conference on Decision and Control, Phoenix, AZ, USA, August 1999.

[21] H. Noura, D. Theilliol, and D. Sauter, "Actuator fault-tolerant control design: demonstration on a three-tank-system," International Journal of Systems Science, vol. 31, no. 9, pp. 1143-1155, 2000.

[22] L. F. Mendoca, J. M. C Sousa, and J. M. G. Sa Da Costa, "Fault tolerant control of a three tank Benchmark using weigted predictive control," in Proceedings of the Foundations of Fuzzy Logic and Soft Computing, 12th International Fuzzy Systems Association World Congress, IFSA 2007, vol. 4529, pp. 732742, Cancun, Mexico, June 2007.

[23] N. Orani, A. Pisano, and E. Usai, "Fault detection and reconstruction for a three-tank system via high-order slidingmode observer," in Proceedings of the 18th IEEE International Conference on Control Applications Parts of 2009 IEEE MultiConference on Systems and Control Saint Petersburg, pp. 1714-1719, Saint Petersburg, Russia, July 2009. 
[24] D. Theilliol, H. Noura, and J.-C. Ponsart, "Fault diagnosis and accommodation of a three-tank system based on analytical redundancy," ISA Transactions, vol. 41, no. 3, pp. 365-382, 2002.

[25] M. Amor, T. Ladhari, S. Hadj Said, and F. M'Sahli, "Hardware implementation of actuator fault estimation applied to a hydrographic plant," in Proceedings of the 19th International Conference on Sciences and Techniques of Automatic Control and Computer Engineering (STA), Sousse, Tunisia, March 2019.

[26] M. Amor, T. Ladhari, S. Hadj Said, and F. M'Sahli, "Actuator fault-tolerant control applied to three-tank system," Mathematical Problems in Engineering, vol. 2020, Article ID 8514049, 9 pages, 2020.

[27] D. H. Zhou, G. Z. Wang, and S. X. Ding, "Sensor fault tolerant control of nonlinear systems with application to a three-tank-system," IFAC Proceedings Volumes, vol. 33, no. 11, pp. 789-794, 2000.

[28] Y. Yang, L. Li, and S. X Ding, "A control-theoretic study on Runge-Kutta methods with application to real-time faulttolerant control of nonlinear systems," IEEE Transactions on Industrial Electronics, vol. 62, no. 6, pp. 3914-3922, 2015.

[29] M. Amor, T. Ladhari, S. Hadj Said, and F. M'Sahli, "On-line continuous-discrete time observer for three-tank system," in Proceedings of the 15th International Multi-Conference on Systems, Signals \& Devices (SSD), Hammamet, Tunisia, March 2018.

[30] M. Hou, Y. S. Xiong, and R. J. Patton, “Observing a three-tank system," IEEE Transactions on Control Systems Technology, vol. 13, no. 3, pp. 478-484, 2005.

[31] M. Hou, Y. S. Xiong, and R. J. Patton, Observer Design for a Three Tank System, The 15thTriennial Word Congress, Barcelona, Spain, 2002. 